\title{
Comparison of Blood Toluene Levels after Inhalation and Oral Administration ${ }^{1}$
}

\author{
Michael J. Sullivan ${ }^{*},{ }^{2}$ ANd RoRy B. CONOLly ${ }^{1,3}$ \\ * Kresge Hearing Research Institute, +Toxicology Program, School of Public Health, \\ The University of Michigan, Ann Arbor, Michigan 48109
}

Received October 2, 1986

\begin{abstract}
The purpose of this investigation was to compare blood toluene levels in SpragueDawley rats after oral and inhalation administration. Groups of 30 rats were dosed by gavage with $86.7,217,433$, or $867 \mathrm{mg}$ toluene $/ \mathrm{kg}$ body wt or exposed for up to $6 \mathrm{hr}, 5$ rats per exposure, to an atmosphere of either 200 or $1000 \mathrm{ppm}$ toluene. Blood was sampled by cardiac puncture from 5 rats in each of the six dose groups at $0.5,1.0,2.0,4.0,6.0$, and 24.0 hr after gavage dosing or the beginning of the inhalation exposure. Blood toluene levels were analyzed. A four-parameter model was fitted to the blood toluene levels of the orally dosed rats. The area under the curve generated by this model, representing total blood toluene concentration over $6 \mathrm{hr}$, was calculated and compared to the area under the blood toluene curve for the 6 -hr inhalation exposure. Integrated areas from the two routes of exposure were used for direct comparison of oral and inhalation exposures. The data demonstrate that gavage dosing can be used to approximate inhalation exposure to toluene. 1988 Academic Press, Inc.
\end{abstract}

\section{INTRODUCTION}

Human exposure to toluene results from its use in the industrial setting and from substance abuse (glue sniffing). These exposures have resulted in numerous effects including altered liver function, CNS changes (Hayden et al., 1977), peripheral neuropathies (Faillace and Guynn, 1976), and renal damage (O'Brien et al., 1971). In our laboratory, toluene given orally has been shown to cause inner ear sensory hair cell lesions and elevated brainstem auditory evoked response (BAER) thresholds in the mid-frequency regions of the cochlea corresponding to $2-8 \mathrm{kHz}$ (Sullivan, 1986). In contrast to our results, studies performed by Pryor et al. (1984) have shown that rats exposed to toluene via inhalation exhibited high-frequency hearing loss. The purpose of this investigation was to determine whether gavage dosing results in blood levels of toluene similar to those obtained during inhalation exposure. These two routes of toluene administration were investigated in order to compare the results of experiments on toluene-induced ototoxicity from the oral and inhalation routes.

\footnotetext{
${ }^{1}$ Supported by The American Petroleum Institute.

2 Present address: NCASI, 260 Madison Ave., No. 1105, New York, NY 10016.

${ }^{3}$ Present address: Northrup Services Inc., Environmental Sciences, 101 Woodman Dr., Suite 12, Dayton, $\mathrm{OH} 45431$
} 


\section{MATERIALS AND METHODS}

Chemicals. Analytical grade toluene, $p$-xylene, and carbon disulfide were purchased from Fisher Scientific Co. (Detroit, MI).

Animals. A total of 210 male Sprague-Dawley rats (Charles River Laboratories, Inc., Portage, MI) were housed four per cage in metal cages and given water and rodent chow (Ralston-Purina Co.) ad libitum. The rats were allowed to become acclimated for at least 10 days before use. The average body weight for the rats at the time of use was $243 \pm 9 \mathrm{~g}$ (SD).

Exposures. Groups of 30 rats were dosed by gavage with $86.7,217,433$, or 867 $\mathrm{mg}(0.10,0.25,0.50$, or $1.0 \mathrm{ml})$ toluene $/ \mathrm{kg}$ body wt or exposed for up to $6 \mathrm{hr}$ to a toluene atmosphere of either 200 or $1000 \mathrm{ppm}\left(3.76 \mathrm{mg}\right.$ toluene $/ \mathrm{m}^{3}$ air). Whole body inhalation exposures, 5 rats per concentration per exposure duration, were in 30-liter positive pressure exposure chambers. Chamber toluene concentrations were calibrated by sampling $1.0 \mathrm{ml}$ of chamber air with a gas-tight syringe both before and during exposures and analyzing for toluene using the gas chromatographic method described below. Toluene atmosphere was generated by passing metered dilution air through a heated flask into which there was metered delivery of toluene liquid. The temperature of the chamber was monitored and used to calculate toluene concentrations. For gavage dosing, toluene was dissolved in corn oil and each treatment group received a total of $1.0 \mathrm{ml}$ of dosing solution $/ \mathrm{kg}$ body wt. Blood was sampled by cardiac puncture from 5 rats in each of the six dose groups at $0.5,1.0,2.0,4.0,6.0$, and $24.0 \mathrm{hr}$ after gavage dose or the beginning of the inhalation exposure, for a total of 30 rats per dose group. An additional 30 rats, 5 per time point, served as controls.

Toluene analysis. Toluene blood levels were analyzed using a method similar to that reported by Benignus et al. (1984b). Two 1.0-ml aliquots of blood from each rat were immediately added to $0.5 \mathrm{ml}$ distilled/deionized water, vortexed, extracted with $1.0 \mathrm{ml}$ of $\mathrm{CS}_{2}$ containing a $10-\mathrm{ppm}$ internal $p$-xylene standard, and vortexed again. The carbon disulfide layer was transferred by pipet into a capped 3.0 glass vial and stored at $4^{\circ} \mathrm{C}$ until analysis. Blood toluene levels were analyzed by injecting an 2.0- $\mu$ l aliquot of $\mathrm{CS}_{2}$ into a Varian Model 3700 gas chromatograph fitted with a FID detector. The 6 foot $\times 1 / 4$ in. i.d. glass column was packed with $5 \%$ Bentone-34 and di-isodedocyl phthalate on $60 / 80$ mesh diatoport solid support. The carrier gas was $\mathrm{N}_{2}$ at $20 \mathrm{ml} / \mathrm{min}$. The column temperature was $100^{\circ} \mathrm{C}$ and the injector and detector temperatures were $220^{\circ} \mathrm{C}$. The attenuation settings were $2 \times 10^{-10}$ and the chart speed was $1 \mathrm{~cm} / \mathrm{min}$. At these conditions, $p$-xylene and toluene had retention times of 2.8 and $4.8 \mathrm{~min}$, respectively. Individual blood levels, ppm (mg/liter), were calculated using the ratio of the internal standard 10 ppm $p$-xylene peak to the toluene peak. Final concentrations are reported as the average of the two samples. The limit of detection was $0.01 \mathrm{ppm}$. Method validation included the analysis of both spiked and unspiked control blood to quantitate recovery and any background interferences. Any samples for which internal xylene recovery was below $60 \%$, as determined by standard calibration curves, were discarded. 


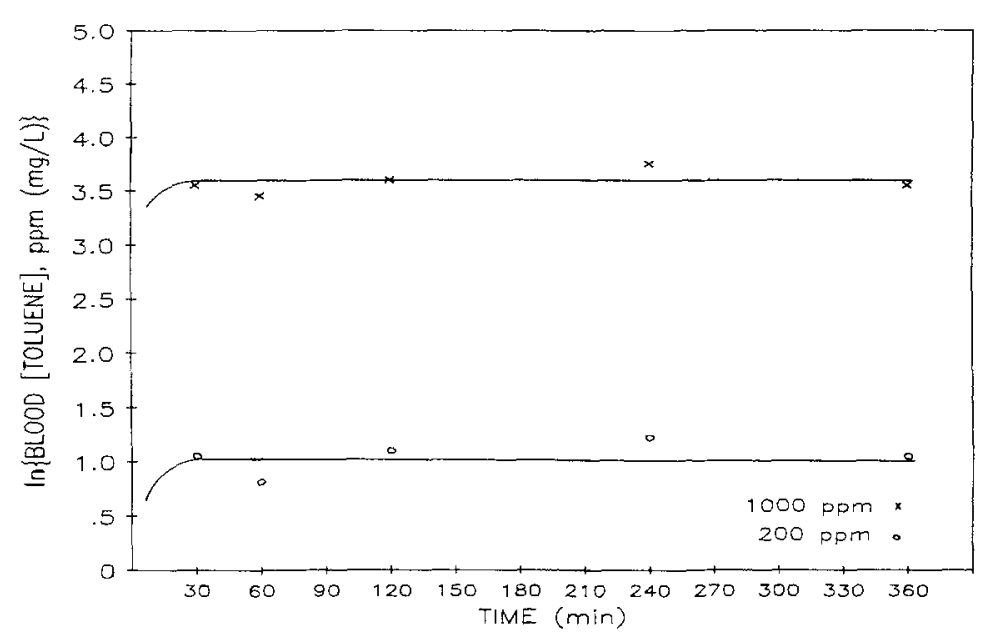

FIG. 1. Blood toluene concentration measured during $6-\mathrm{hr}$ inhalation exposure to 200 or $1000 \mathrm{ppm}$.

Modeling. The toluene blood levels from the orally dosed rats were fitted to the four-parameter (P1, P2, P3, and P4) model,

$$
\begin{aligned}
\ln (\text { blood toluene }, \mathrm{ppm})= & (\ln \mathrm{P} 1)(\text { In dose }, \mathrm{mg} / \mathrm{kg}) \\
& +\ln (1-e(-\mathrm{P} 2(\mathrm{time}, \mathrm{min})) \\
& -(\mathrm{P} 3-(\mathrm{P} 4(\mathrm{ln} \text { dose }, \mathrm{mg} / \mathrm{kg})))
\end{aligned}
$$

(Benignus et al., 1984a), using the BMDP statistical package for nonlinear regression (Jennrich and Ralston, 1983). The area under the curves generated by this model, representing total blood toluene concentration over $6 \mathrm{hr}$ from oral dosing,

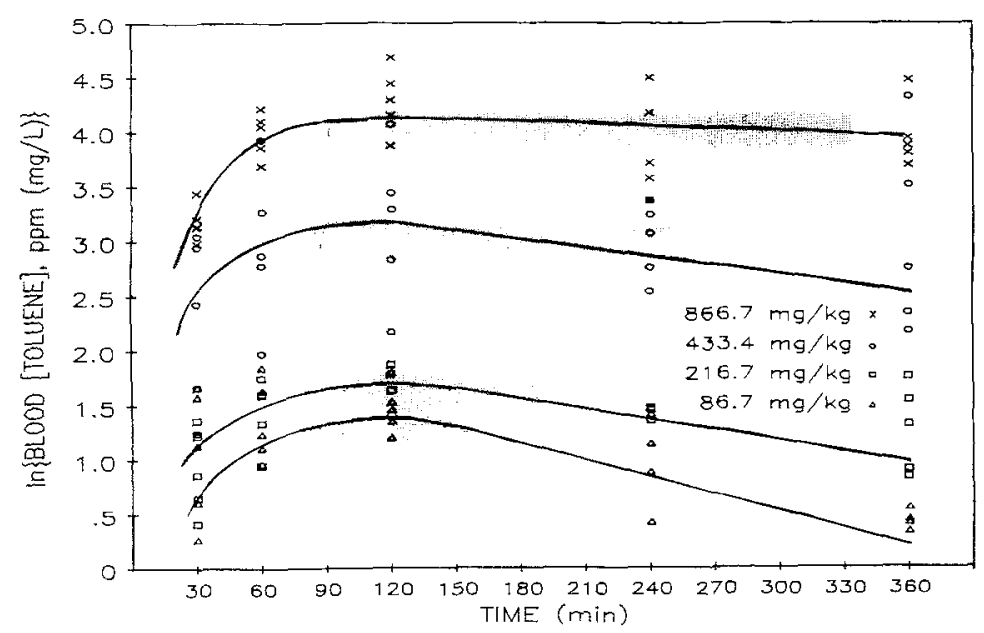

FIG. 2. Blood toluene concentrations measured over 6 hr after gavage dosing with $86.7,217,433$, or $867 \mathrm{mg}$ toluene/kg body wt. Shaded areas of each curve indicate the durations of the $95 \%$ maximum toluene blood concentration for that dose. 


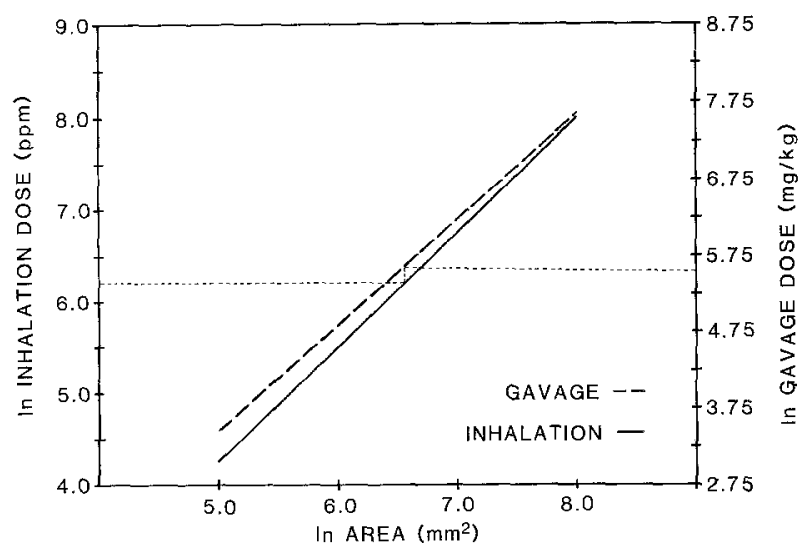

FIG. 3. Plot of both $\ln$ (gavage dose) [Eq. (4)] and $\ln ($ inhalation exposure level) [Eq. (2)] against $\ln$ (calculated area under each respective curve). Dotted line shows comparison of 500 ppm inhalation exposure to $230 \mathrm{mg} / \mathrm{kg}$ gavage dose.

was calculated by integration of the curves and compared to the area under the blood toluene curves for the 6-hr inhalation exposures. Integrated areas from the two routes of exposure were used for direct comparison of oral $(\mathrm{mg} / \mathrm{kg})$ and inhalation ( $\mathrm{ppm})$ exposures. The intervals during which the blood toluene concentrations were at $\geqslant 95 \%$ of their maximum level were calculated from the gavage dose data and compared to the inhalation generated steady-state blood toluene levels.

\section{RESULTS}

Toluene blood levels during the 6-hr inhalation exposures were consistent with the toluene blood level-inhalation concentration dose relationship reported by Benignus et al. (1984b). The data in Fig. 1 show that the steady-state toluene blood concentrations for the 200 and $1000 \mathrm{ppm}\left(3.76 \mathrm{mg}\right.$ toluene $/ \mathrm{m}^{3}$ air) inhalation exposures were 2.89 and $37.23 \mathrm{ppm}$ (mg/liter), respectively. Steady-state blood

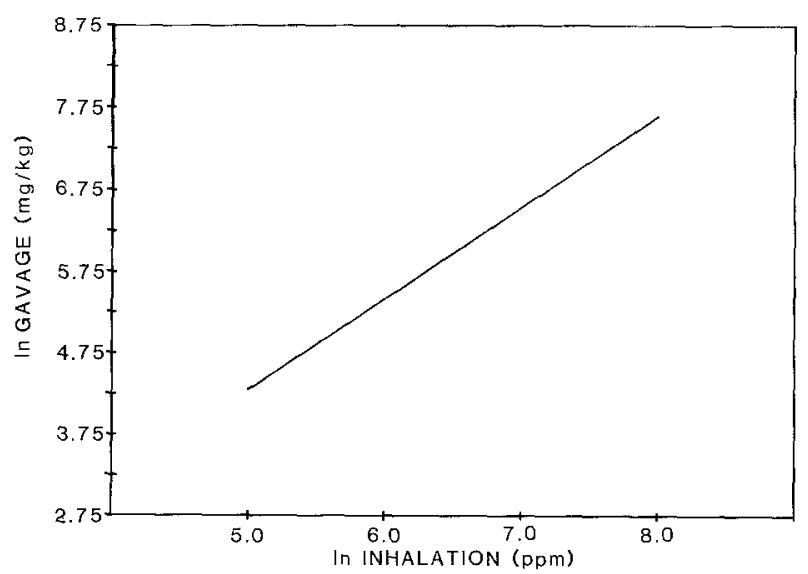

FIG. 4. Plot of $\ln$ (gavage dose) against $\ln$ (inhalation exposure level) [Eq. (5)]. 
concentrations were reached in both exposure levels within $30 \mathrm{~min}$. Plotting In(calculated area under these two curves) against $\ln$ (inhalation dose, ppm) results in the inhalation line in Fig. 3 described by the equation

$$
\ln (\text { inhalation dose, } \mathrm{ppm})=(1.901 \ln (\text { area }))-0.785 \text {. }
$$

The toluene blood levels from the four gavage dose levels are shown in Fig. 2 (symbols) along with the model-generated predicted values (curves). Nonlinear regression analysis (Jennrich and Ralston, 1983) of the data results in the following equation based on the model in Eq. (1):

$$
\begin{aligned}
\ln (\text { blood toluene, } \mathrm{ppm})= & (\ln 2.78)(\ln \text { dose }, \mathrm{mg} / \mathrm{kg}) \\
& +\ln (1-e(-0.008(\text { time }, \mathrm{min})) \\
& -(0.015-(0.0015(\ln \text { dose }, \mathrm{mg} / \mathrm{kg}))) .
\end{aligned}
$$

The $\mathrm{R}^{2}$ for Eq. (3) using the data from all four dose levels is 0.80 . Plotting the $\ln$ (calculated area under these four curves) against the $\ln ($ oral dose $(\mathrm{mg} / \mathrm{kg})$ ) results in the gavage line in Fig. 3 described by the equation

$$
\text { In dose }(\mathrm{mg} / \mathrm{kg})=1.386(\ln \text { area })-10.22 \text {. }
$$

\section{DISCUSSION}

The 6-hr blood profiles from the oral toluene doses (Fig. 2) show several patterns. The peak blood toluene concentration increases with increasing dose. The shape of the curve flattens out with increasing dose, as indicated by lengthening durations of $95 \%$ maximum concentration times. The data show that blood profiles from higher oral doses of 433 and $867 \mathrm{mg}$ toluene/ $\mathrm{kg}$ body wt more closely approximate the steady-state inhalation blood profiles than do the lower oral doses of 86.7 and $217 \mathrm{mg}$ toluene/ $\mathrm{kg}$ body wt. At $24 \mathrm{hr}$, blood toluene levels from all doses by both routes are at or below minimal detection levels. This indicates that toluene carryover from day to day is minimal.

Figure 3 allows direct comparison of toluene blood levels after 6-hr inhalation exposure and after gavage dosing. For example, a 6-hr $500 \mathrm{ppm}$ toluene exposure

\begin{tabular}{|c|c|c|c|c|c|c|}
\hline & \multicolumn{4}{|c|}{ Parameters $^{a}$} & \multicolumn{2}{|c|}{$R^{2 b}$} \\
\hline & Pl & $\mathrm{P} 2$ & P3 & P4 & Gavage & Subcutaneous \\
\hline $1^{c}$ & 2.78 & 0.008 & 0.015 & 0.0015 & 0.80 & N/A \\
\hline $2^{d}$ & 1.58 & 0.016 & 0.008 & 0.0012 & 0.58 & 0.74 \\
\hline
\end{tabular}
has a blood toluene $x$ time level equivalent to that of a $230 \mathrm{mg}$ toluene $/ \mathrm{kg}$ body wt dose. Combining Eqs. (2) and (4) by first solving for $\ln ($ area) and then equating

TABLE !

Model Parameters and $R^{2}$ Values For Gavage and Subcutaneous Dosing

\footnotetext{
a Parameters (P1, P2, P3, and P4) from Eq. (1).

${ }^{b} R^{2}$ is the measure of fit of data to model.

$c$ Gavage dosing (this experiment).

${ }^{d}$ Subcutaneous dosing (Benignus et al., 1984a).
} 
generates the following equation (Fig. 4), which describes the relationship between the two routes:

$$
\ln (\text { gavage dose, } \mathrm{mg} / \mathrm{kg})=1.27 \ln (\text { inhalation dose, } \mathrm{ppm})-9.22 \text {. }
$$

The parameters in Eq. (3), which describe orally dosed toluene blood levels, can be compared to those reported for subcutaneous toluene dosing (Benginus et al., 1984). Table 1 shows the values of the four parameters and the respective $R^{2}$ values for both oral and subcutaneous dosing. P1, describes the effect of the toluene dose level on the blood toluene level or the nonlinearlity of blood levels with increasing dose. P1 is greater for the oral route indicating a more linear effect of increasing dose on increasing blood concentration. P2 is a measure of uptake; P3 and $\mathrm{P} 4$ are measures of elimination. The values for the parameters indicate that uptake is slower (P2) and elimination is faster (P3) for the gavage dosing compared to subcutaneous dosing. However, P4 (elimination) is not different between the two routes. When the blood toluene data from gavage dosing is fit to the model for subcutaneous dosing, $R^{2}$ decreases from 0.80 to 0.58 . This suggests that the two routes (oral and subcutaneous) produce different blood toluene profiles.

The results of this investigation show that a gavage dose of toluene which approximates inhalation blood toluene levels can be calculated using Eq. (5). Detailed analyses of the partition of toluene into various compartments, metabolism and elimination, as are done in pharmacokinetic studies, were not performed here. However, while elimination pathways would be the same for the two exposure routes, the data show that the rates of elimination were different. This is supported by the finding that $\mathrm{P} 3$, an elimination parameter in the blood profile model, is different for oral and subcutaneous routes. As the gavage dose increases, the plateau phase of the blood-time concentration profiles from the gavage doses lengthen and better approximate the steady-state blood toluene concentration during inhalation exposure. This limits the applicability of approximating low-level inhalation of toluene with oral toluene administration. The gavage route has slower uptake and faster elimination than the subcutaneous route, which therefore has longer plateau phases than gavage dosing. This indicates that blood toluene levels after subcutaneous injection more closely approximate blood levels during inhalation than do blood levels after gavage. This study shows that blood concentrations of toluene similar to those generated by a 6 -hr inhalation exposure to $1000 \mathrm{ppm}$ toluene can be achieved by gavage dosing.

\section{REFERENCES}

Benignus, V. A., Muller, K. E., Barton, C. N., and Bittikofer, J. A. (1984a). Toluene blood levels following sub-cutaneous injection of toluene in the rat. Environ. Res. 33, 441-453.

Benignus, V. A., Muller, K. E., Graham, J. A., and Barton, C. N. (1984b). Toluene levels in blood and brain of rats as a function of toluene level in inspired air. Environ. Res. 33, 39-46.

Faillace, L. A., and Guynn, R. W. (1976). Abuse of organic solvents. Physiosomatics 17, 188-198.

Hayden, J. W., Peterson, R. G., and Bruckner, J. V. (1977). Toxicology of toluene(methylbenzene): Review of current literature. Clin. Toxicol. 11(5), 549-559.

Jennrich, R. I., and Ralston, M. (1983). Non-linear regression and derivative free non-linear regres- 
sion. In "BMDP Statistical Software" (W. J. Dixon, Ed.), pp. 290-314. Univ. of California Press, Berkeley.

O'Brien, E. T., Yeoman, W. B., and Hobby, J. A. E. (1971). Hepatorenal damage from toluene in a "glue-sniffer." Brit. Med. J. 2, 29-30.

Pryor, G. T., Rebert, C. S., Dickerson, J., and Feeney, E. M. (1984). Factors affecting toluene-induced ototoxicity in rats. Neurobehav. Toxicol. Teratol. 6, 223-238.

Sullivan, M. J. (1986). "Ototoxicity of Toluene in Rats." Doctoral Thesis, University of Michigan Microfilms, Ann Arbor. 